\title{
NAFLD Prevalence in a Young Cadaveric Organ Donor Population
}

\author{
Ali Jafarian ${ }^{1,}$; Amirpasha Ebrahimi ${ }^{1}$; Farid Azmoudeh Ardalan ${ }^{2}$; Habibollah Dashti ${ }^{1}$; \\ Mojgan Rahimi ${ }^{3}$; Mandana Salehi ${ }^{1}$; Mohsen Nasiri Toosi ${ }^{4}$ \\ ${ }_{1}^{1}$ Hepatobiliary and Liver Transplantation Division, Department of General Surgery, Imam-Khomeini Hospital, Tehran University of Medical Sciences (TUMS), Tehran, IR Iran \\ 2 Department of Pathology, Imam-Khomeini Hospital, Tehran University of Medical Sciences (TUMS), Tehran, IR Iran \\ 3 Department of Anesthesiology and Intensive Care, Imam-Khomeini Hospital, Tehran University of Medical Sciences (TUMS), Tehran, IR Iran \\ ${ }^{4}$ Department of Anesthesiology and Intensive Care, Imam-Khomeini Hospital, Tehran University of Medical Sciences (TUMS), Tehran, IR Iran
${ }_{\text {Gastroenterology Division, Internal Medicine Department, Imam-Khomeini Hospital, Tehran University of Medical Sciences (TUMS), Tehran, IR Iran }}$ \\ ${ }^{*}$ Corresponding Author: Ali Jafarian, Hepatobiliary and Liver Transplantation Division, Department of General Surgery, Imam-Khomeini Hospital, Tehran University of Medical Sci- \\ ences (TUMS), Tehran, IR Iran. Tel/Fax: +98-2166581657, E-mail: jafarian@tums.ac.ir
}

Received: June 28, 2014; Revised: September 4, 2014; Accepted: September 11, 2014

\begin{abstract}
Background: Liver transplantation is a critical survival point for patients with end stage liver diseases. It can dramatically increase patients' survival if the donor liver is intact. One aspect of liver health is absence of steatosis. Nonalcoholic Steato Hepatitis (NASH) and Nonalcoholic Fatty Liver Disease (NAFLD) are increasing among young adults and patients living with chronic liver diseases.

Objectives: In this study, we determined the prevalence of NALFD in livers of brain-dead donors in Imam-Khomeini hospital Complex, Tehran, Iran. We assumed that the calculated prevalence would represent NAFLD prevalence in Iranian population in the age range of 20-60 years.

Materials and Methods: All eligible brain dead liver transplant donors were enrolled in the survey from March 21, 2011 to March 21, 2013 in Imam-Khomeini hospital Complex. Eligible participants were donors aged 20 to 60 years without any obvious history of liver disease. Liver needle biopsy was performed at the end of the transplant operation; time zero biopsy. We calculated the prevalence of NAFLD among brain-dead donors. Moreover, the frequency of NASH was calculated based on the NAS (NAFLD Activity Score).

Results: Among 116 cases, two were diagnosed as probable NASH. There was a significant association between NAFLD and male gender (P $=0.04)$. Moreover, we found a higher steatosis level in male gender. There was a significant association between NAFLD and BMI $(P=0.05)$. Those with BMI more than 27 had severe steatosis.

Conclusions: Our comprehensive literature review showed that our study was the first investigation in Iran and the region, which determined the prevalence of NAFLD based on tissue diagnosis. We believe that the prevalence of NAFLD/NASH in our donors can represent the overall prevalence in this age group in Iran.
\end{abstract}

Keywords: Steatosis; NASH; NAFLD; Prevalence; Cadaver

\section{Background}

Liver transplantation is a critical survival point for patients with end stage liver diseases. It can dramatically increase patients' survival if the donor liver is intact. One aspect of liver health is absence of steatosis. Fatty liver refers to accumulation of fat droplet, dominantly triglyceride in the liver either macro-vesicular or micro-vesicular. Based on other classification, fat accumulation can be due to either alcohol or not (1). Nonalcoholic Steato Hepatitis (NASH) and Nonalcoholic Fatty Liver Disease (NAFLD) show predominantly macro-vesicular type of steatosis. This category of fatty liver disease translocates cellular organelle, but does not interfere with cellular functions. This type is increasing among young adults and patients living with chronic liver diseases. Conversely, micro-vesicular type does not translocate cellular organelles, but interfere with normal cellular biology (1-5). Predisposing factors of NASH include 20-60 years of age, female gender, BMI > 30, diabetes mellitus, consuming some drugs (e.g. steroids and methotrexate), insulin resistance status, ab- dominal surgery (e.g. gastric surgery, small intestine bypass or ileostomy), and metabolic associated factors (e.g. rapid weight loss, long time fasting and TPN therapy) (35). Because the disease prevalence is increasing, it is very important to estimate it. Although definite diagnosis of NASH and NAFLD is based on histopathology findings of liver biopsy, it is not ethical to biopsy normal population to obtain the prevalence of NASH (2-5).

\section{Objectives}

In this study, we determined the frequency of NALFD in livers of brain-dead donors in Imam-Khomeini hospital Complex, Tehran, Iran. We assumed that the calculated prevalence would represent NAFLD prevalence in Iranian population in the age range of 20-60 years. To the best of our knowledge, this was the first study in Iran and the Middle East to investigate the prevalence of NAFLD and $\mathrm{NASH}$, based on tissue diagnosis.

Copyright (C) 2014, Kowsar:; Published by Kowsar. This is an open-access article distributed under the terms of the Creative Commons Attribution-NonCommercial 4.0 International License (http://creativecommons.org/licenses/by-nc/4.0/) which permits copy and redistribute the material just in noncommercial usages, provided the original work is properly cited. 


\section{Materials and Methods}

In this cross-sectional, retrospective study, all eligible brain dead liver transplant donors were enrolled in the survey from March 21, 2011 to March 21, 2013 in Imam-Khomeini hospital Complex. Eligible participants were donors aged 20 to 60 years, without any obvious history of liver disease, alcohol and drug abuse. Their medical and family histories were unremarkable. Liver needle biopsy was performed at the end of the transplant operation; time zero biopsy. Specimens were fixed in $10 \%$ buffered formalin overnight and submitted for histopathology evaluation. After grossing of the specimens, tissues were processed and paraffin embedded. H\&E, Masson's trichrome and reticulin-stained slides were evaluated for adequacy and other findings by one pathologist. Specimens with more than four portal tracts were considered adequate. At last, we calculated the prevalence of NAFLD among brain-dead donors. Furthermore, the frequency of NASH was calculated based on the NAS (NAFLD Activity Score). All transplanted livers were examined by pathology. Health status of all subjects was documented before the transplantation. Unhealthy liver specimens either macro-structural or histological were excluded. Additionally, NAFLD associated anthropometric indices including age, gender and BMI were collected from donor data registration. The prevalence of NAFLD and NASH (based on the NAS) was calculated. Data was analyzed using SPSS software package version 22.0. Descriptive statistics and Mann-Whitney U test were used. P value $<0.05$ was considered significant.

\subsection{Ethical Considerations}

This investigation was confirmed by our ethical committee of Imam Khomeini Hospital Complex, Tehran University of Medical Sciences, Tehran, Iran. We obtained verbal and written consents from all next-of-kin of eligible donors.

\section{Results}

One hundred and sixty eligible donors were enrolled in the study. We described three variables as age, gender and BMI. Moreover, we estimated NAFLD and (probable) NASH prevalence. At last, we evaluated any potential association between variables and NAFLD prevalence.

\subsection{Descriptive Studies}

Totally, 87 cases were male and 29 females. The mean age was 33 years. The mean BMI was $27 \mathrm{~kg} / \mathrm{m}^{2}$. Based on the presence of fatty change, cases were collected into two major groups. Group one NAFLD positive and group two NAFLD negative. In each group age, gender and BMI were evaluated separately either by descriptive or analytic tests. Table 1 showed descriptive analysis in each group.

Fifteen cases (13\%) had steatosis in the liver biopsy (positive NAFLD) (Figure 1). According to the NAS scoring, only two cases were suggestive of NASH (probable NASH). The first case score was four; moderate steatosis $(+2)$ and lobular inflammation (2-4 foci per $200 x$ field, +2 ). The second case score was three; mild steatosis $(+1)$ and lobular inflammation (2-4 foci per 200x field, +2$)$. The degree of steatosis was determined in three levels. Mild level was $5-33 \%$ steatosis, moderate level as 33-66\% and more than $66 \%$ of steatosis as severe. Moreover, steatosis less than $5 \%$ was neglected. Table 2 showed distribution of steatosis levels among with NAFLD.

There was a significant association between NAFLD and gender ( $P$ value: 0.04). Spearman correlation showed a positive association between NAFLD and gender (r: 0.6). In our study, being male increased the probability of NAFLD and steatosis level. Besides, we found a significant association between NAFLD and BMI (P value $=0.05$ ). Those with overweigh BMI had severe level of steatosis. Regarding BMI level distribution in positive NAFLD group, four (26.7\%) patients had normal BMI, while 8 (53.3\%), 3 (20\%) and 0 patients were overweight, obese and morbid obese, respectively. We did not find any considerable association between steatosis and age. One-way ANOVA test showed a P value of 0.8 .

\begin{tabular}{lccc}
\hline \multicolumn{4}{l}{ Table 1. Descriptive Reports of Each Group } \\
\hline Mean & ${\text { Age, } \mathbf{y}^{\mathrm{a}}}^{\mathrm{a}}$ & Gender & BMI $^{\mathrm{a}}$ \\
\hline NAFLD positive (15) & 36 & 12 Males, 3 Females & 28 \\
NAFLD negative (101) & 30 & 75 Males, 26 Females & 24 \\
Total & 33 & 87 Males, 29 Females & 27 \\
\hline
\end{tabular}

a Data are present as Mean+/-SD.

\begin{tabular}{lcc}
\hline Table 2. Distribution of Steatosis Level & \\
\hline Grade & Frequency & Percent \\
\hline Mild (5-33\%) & 12 & 80.0 \\
Mod (33-66\%) & 2 & 13.3 \\
Severe (>66\%) & 1 & 6.7 \\
Total & 15 & 100.0 \\
\hline
\end{tabular}

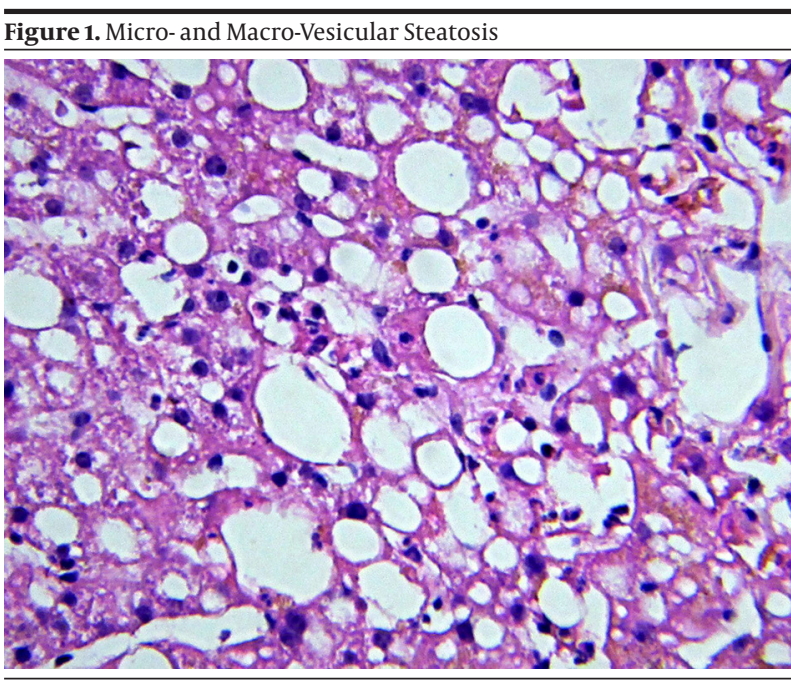

Ballooning degeneration and neutrophil infiltration are seen. H\&E staining. 


\section{Discussion}

In this study, the prevalence of NAFLD was estimated as $13 \%$. Most subjects ( $80 \%$ ) had mild steatosis, while moderate and severe steatosis were $13 \%$ and $7 \%$, respectively. Among 116 cases, two were diagnosed as probable NASH. There was a significant association between NAFLD and male gender $(\mathrm{P}=0.04)$. Furthermore, we found a higher steatosis level in male gender. There was a significant association between NAFLD and BMI $(\mathrm{P}=0.05)$. Those with BMI more than 27 had a severe level of steatosis. The frequency of NASH was calculated based on the NAS (NAFLD Activity Score).

NAS has been designed and validated by the Pathology Committee of the NASH Clinical Research Network. NAS describes a histological scoring system, which discourses the full spectrum of NAFLD lesions and gives an activity score for NAFLD. NAS contains 14 histological features, four of which are evaluated semi-quantitatively as: steatosis (0-3), lobular inflammation (0-2), hepatocellular ballooning (0-2), and fibrosis (0-4). Other features are recorded as present or absent $(1,5-7)$. Steatosis is one of the most important parameters in evaluation of acceptability of the liver graft for transplantation. Normally, livers with more than $60 \%$ of steatosis are unacceptable for transplantation (3-5). Fortunately, this level of steatosis was not very common in our study population. Review of the literature showed that NAFLD and NASH are more common in middle-aged women, those with higher BMI level, intestinal and gastric surgeries and individuals who consume cytotoxic drugs like methotrexate (1-10). Farrell et al. defined the difference between NASH and NAFLD; they stated that NASH encompasses a pathogenic link in the chain of nonalcoholic fatty liver diseases (NAFLD) that spreads from mild steatosis to some cases of cryptogenic cirrhosis (1).

Based on the Third National Health and Nutritional Examination Survey (NHANES III), 3\% to 23\% of adults had NAFLD/NASH (2-4). In studies that gathered data based on autopsy or biopsy findings, about 70\% of obese people had hepatic steatosis and/or raised level of alanine aminotransferase (ALT) (4-10); 20\% of those with hepatic steatosis presented NASH evidences $(11,12)$. Other studies mentioned that around $10 \%$ of patients with diabetes had cirrhosis. However, recent studies indicated that both the prevalence and severity of NASH increase considerably in patients with type 2 diabetes $(9,10,13-17)$. These studies indicated that raising trend of NAFLD/NASH is due to high prevalence rates of obesity and type $2 \mathrm{DM}(9,10,13-17)$.

Concerns around NASH outcomes are more than steatosis alone. Actually, $20-25 \%$ of cases with NASH would progress to cirrhosis $(16,18-21)$. There is a growing evidence that a large proportion of 'cryptogenic cirrhosis' may be attributed to NASH $(16,18-21)$. Besides, subacute hepatic failure may be attributed to NASH (22). Previous studies of NAFLD/NASH estimated good overall prognosis $(23,24)$. However, recent studies have distinct cases according to fibrotic liver leading to poor prognosis of liver failure
$(18,25,26)$. Previous studies mentioned that prevalence of NAFLD/NASH is estimated at about 2\%-10\% in developed countries. The prevalence of NAFLD/NASH in Iranians has been reported $2.9 \%$ to $7.1 \%$ in general population (27-29) and $55.8 \%$ in patients with type 2 diabetes mellitus (30). Factors definitely attributed to pathogenesis of NAFLD/ NASH include: type 2 diabetes mellitus, family history of type 2 diabetes, insulin resistance with or without glucose intolerance, central obesity (waist: hip $>0.85$ in women, $>0.90$ in men; waist $>85 \mathrm{~cm}$ in women, $>97 \mathrm{~cm}$ in men), obesity (BMI $>30 \mathrm{~kg} / \mathrm{m}^{2}$ in white people, $>27 \mathrm{~kg} /$ $\mathrm{m} 2$ in Asians), hypertriglyceridemia, rapid and massive weight loss in overweight subjects. Although definite diagnosis of NAFLD/NASH is based on pathologic findings, routine feasible approach for NAFLD/NASH diagnosis is clinical. Liver function test alongside serum lipid profile and imaging would confirm the diagnosis. Despite the fact, the most accurate and reliable approach to confirm NAFLD/NASH is liver biopsy.

Features of steatohepatitis in liver biopsy include hepatocellular injury (further than simple fatty change), inflammation and fibrosis (31). The most characteristic feature of steatohepatitis is ballooning, typically associated with the formation of Mallory's hyaline. Mallory bodies in NAFLD are often small and poorly formed and may be difficult to detect in routinely stained sections $(32,33)$. In a study performed in Shariati hospital, Tehran, Iran, Sohrabpour et al. analyzed 5589 individuals aged 18 to 65 years. The overall prevalence of presumed NASH was $2.9 \%$. They found that male gender, urban lifestyle, and being overweight or obese were significantly associated with presumed NASH (29). These finding are relevant to our results, as we noted that male gender and being overweight had significant correlations with NAFLD/NASH prevalence. Although Sohrabpour et al. investigated a large population, they did not consider pathologic findings for their assessment (29).

They defined "presumed NASH" individuals as those with elevated serum ALT level (ALT $\geq 40 \mathrm{IU} / \mathrm{L}$ ) with no history of alcohol consumption and negative results for HBV and HCV infection.

In another report from Shiraz, southern Iran during a 10-month period, cluster random sampling of Iranian general population was performed in Shiraz region. Bagheri-Lankarani et al. performed clinical-laboratory approaches to estimate the prevalence of NAFLD. NAFLD was diagnosed in 176 (21.5\%) cases. They found that patients with NAFLD were significantly older $(P<0.001)$, mostly male $(\mathrm{P}=0.004)$ and with higher $\mathrm{BMI}(\mathrm{P}<0.001)$. They also had higher prevalence of hypertension $(\mathrm{P}<$ 0.001), high FBS level $(\mathrm{P}<0.001)$, high cholesterol $(\mathrm{P}=$ $0.026)$, high triglyceride $(\mathrm{P}<0.001)$ and high waist circumference $(\mathrm{P}<0.001)$. They concluded that NAFLD occurred significantly with higher prevalence in those with metabolic syndrome, compared to healthy subjects ( $\mathrm{P}<$ 0.001) (33). Their anthropometric findings were the same as ours. Nonetheless, they did not consider pathologic 
findings in their study $(33,34)$. Our comprehensive literature review showed that our study was the first study in Iran and the region, which determined the prevalence of NAFLD based on tissue diagnosis. We believe that obtained prevalence of NAFLD/NASH in our donors can represent the overall prevalence in this age group in Iran.

\subsection{Study Limitations}

Our study had some limitations. First and the most important, our results could underestimate the true prevalence of NAFLD/NASH in our country, because patients with overt steatosis may not be enrolled for transplantation and their livers are rejected based on macroscopic findings and without histopathologic evaluation. The other limitation of our survey was our small sample size.

\section{Authors' Contributions}

Design idea: Ali Jafarian and Amirpasha Ebrahimi; data gathering and statistical analysis: Farid Azmoudeh Ardalan and Mohsen Nasiri Toosi; manuscript preparation: Amirpasha Ebrahimi, Mojgan Rahimi, Mandana Salehi and Habibollah Dashti.

\section{References}

1. Farrell GC, George J, Hall PDLM, McCullough AJ. Fatty liver disease.: John Wiley and Sons; 2008.

2. Mofrad P, Contos MJ, Haque M, Sargeant C, Fisher RA, Luketic VA, et al. Clinical and histologic spectrum of nonalcoholic fatty liver disease associated with normal ALT values. Hepatology. 2003;37(6):1286-92.

3. Hui JM, Farrell GC, Kench JG, George J. High sensitivity C-reactive protein values do not reliably predict the severity of histological changes in NAFLD. Hepatology. 2004;39(5):1458-9.

4. Loria P, Lonardo A, Leonardi F, Fontana C, Carulli L, Verrone AM, et al. Non-organ-specific autoantibodies in nonalcoholic fatty liver disease: prevalence and correlates. Dig Dis Sci.2003;48(11):2173-81.

5. Adams LA, Feldstein A, Lindor KD, Angulo P. Nonalcoholic fatty liver disease among patients with hypothalamic and pituitary dysfunction. Hepatology. 2004;39(4):909-14.

6. Luef GJ, Waldmann M, Sturm W, Naser A, Trinka E, Unterberger I, et al. Valproate therapy and nonalcoholic fatty liver disease. Ann Neurol. 2004;55(5):729-32.

7. Bellentani S, Tiribelli C. The spectrum of liver disease in the general population: lesson from the Dionysos study. J Hepatol. 2001;35(4):531-7.

8. Bellentani S, Saccoccio G, Costa G, Tiribelli C, Manenti F, Sodde $\mathrm{M}$, et al. Drinking habits as cofactors of risk for alcohol induced liver damage. The Dionysos Study Group. Gut. 1997;41(6):845-50.

9. Hayashi PH, Harrison SA, Torgerson S, Perez TA, Nochajski T, Russell M. Cognitive lifetime drinking history in nonalcoholic fatty liver disease: some cases may be alcohol related. Am J Gastroenterol. 2004;99(1):76-81.

10. Clarkson VC, Hall P,, Shephard E, Kirsch R, Marais D. Ethanol feeding increases CYP2E11 activity in the methionine choline deficient mouse model for NASH (Abstract). Liver Int J. 2004;24(Suppl. 4):18.

11. Laurin J. Motion - all patients with NASH need to have a liver biopsy: arguments against the motion. Can J Gastroenterol. 2002;16(10):722-6.

12. Xu A, Wang Y, Keshaw H, Xu LY, Lam KS, Cooper GJ. The fat-derived hormone adiponectin alleviates alcoholic and nonalcoholic fatty liver diseases in mice. J Clin Invest. 2003;112(1):91-100.

13. Nanji AA. Another animal model for nonalcoholic steatohepatitis: how close to the human condition? Am J Clin Nutr. 2004;79(3):350-1.
14. Lieber CS, Leo MA, Mak KM, Xu Y, Cao Q, Ren C, et al. Model of nonalcoholic steatohepatitis. Am J Clin Nutr. 2004;79(3):502-9.

15. Sanyal AJ, Contos MJ, Sterling RK, Luketic VA, Shiffman ML, Stravitz RT, et al. Nonalcoholic fatty liver disease in patients with hepatitis $\mathrm{C}$ is associated with features of the metabolic syndrome. Am J Gastroenterol. 2003;98(9):2064-71.

16. Lonardo A, Adinolfi LE, Loria P, Carulli N, Ruggiero G, Day CP. Steatosis and hepatitis $C$ virus: mechanisms and significance for hepatic and extrahepatic disease. Gastroenterology. 2004;126(2):586-97.

17. Hui JM, Sud A, Farrell GC, Bandara P, Byth K, Kench JG, et al. Insulin resistance is associated with chronic hepatitis $C$ virus infection and fibrosis progression [corrected]. Gastroenterology. 2003;125(6):1695-704.

18. Hui JM, Hodge A, Farrell GC, Kench JG, Kriketos A, George J. Beyond insulin resistance in NASH: TNF-alpha or adiponectin? Hepatology. 2004;40(1):46-54.

19. Weiss R, Dziura J, Burgert TS, Tamborlane WV, Taksali SE, Yeckel $\mathrm{CW}$, et al. Obesity and the metabolic syndrome in children and adolescents. N Engl J Med. 2004;350(23):2362-74.

20. Wanless IR, Nakashima E, Sherman M. Regression of human cirrhosis. Morphologic features and the genesis of incomplete septal cirrhosis. Arch Pathol Lab Med. 2000;124(11):1599-607.

21. Starkel P, Sempoux C, Leclercq I, Herin M, Deby C, Desager JP, et al. Oxidative stress, KLF6 and transforming growth factor-beta upregulation differentiate non-alcoholic steatohepatitis progressing to fibrosis from uncomplicated steatosis in rats. $J$ Hepatol. 2003;39(4):538-46.

22. Bugianesi E, Manzini P, D'Antico S, Vanni E, Longo F, Leone N, et al. Relative contribution of iron burden, HFE mutations, and insulin resistance to fibrosis in nonalcoholic fatty liver. Hepatology. 2004;39(1):179-87.

23. Lerat H, Honda M, Beard MR, Loesch K, Sun J, Yang Y, et al. Steatosis and liver cancer in transgenic mice expressing the structural and nonstructural proteins of hepatitis C virus. Gastroenterology. 2002;122(2):352-65.

24. Rubbia-Brandt L, Quadri R, Abid K, Giostra E, Male PJ, Mentha G, et al. Hepatocyte steatosis is a cytopathic effect of hepatitis C virus genotype 3. J Hepatol. 2000;33(1):106-15.

25. Castera L, Hezode C, Roudot-Thoraval F, Bastie A, Zafrani ES, Pawlotsky JM, et al. Worsening of steatosis is an independent factor of fibrosis progression in untreated patients with chronic hepatitis C and paired liver biopsies. Gut. 2003;52(2):288-92.

26. Nitecki J, Jackson FW, Allen ML, Farr VL, Jackson FW. Effect of phlebotomy on non-alcoholic steatohepatitis. Gastroenterology .2000;118

27. Alavian SM, Mohammad-Alizadeh AH, Esna-Ashari F, Ardalan G, Hajarizadeh B. Non-alcoholic fatty liver disease prevalence among school-aged children and adolescents in Iran and its association with biochemical and anthropometric measures. Liver Int. 2009;29(2):159-63.

28. Rogha M, Najafi N, Azari A, Kaji M, Pourmoghaddas Z, Rajabi F, et al. Non-alcoholic Steatohepatitis in a Sample of Iranian Adult Population: Age is a Risk Factor. Int J Prev Med. 2011;2(1):24-7.

29. Sohrabpour A, Rezvan H, Amini-Kafiabad S, Dayhim M, Merat S, Pourshams A. Prevalence of Nonalcoholic Steatohepatitis in Iran: A Population based Study. Middle East J Dig Dis. 2010;2(1):14-9.

30. Merat S, Yarahmadi S, Tahaghoghi S, Alizadeh Z. Prevalence of Fatty Liver Disease among Type 2 Diabetes Mellitus Patients and its Relation to Insulin Resistance. Middle East J Dig Dis. 2011;1(2):74-9.

31. Hubscher SG. Histological assessment of non-alcoholic fatty liver disease. Histopathology. 2006;49(5):450-65.

32. Vigano M, Vergani A, Trombini P, Paleari F, Piperno A. Insulin resistance influence iron metabolism and hepatic steatosis in type II diabetes. Gastroenterology. 2000;118(5):986-7.

33. Lankarani KB, Ghaffarpasand F, Mahmoodi M, Lotfi M, Zamiri N Heydari ST, et al. Non alcoholic fatty liver disease in southern Iran: a population based study. Hepat Mon. 2013;13(5).

34. Kleiner DE, Brunt EM, Van Natta M, Behling C, Contos MJ, Cummings OW, et al. Design and validation of a histological scoring system for nonalcoholic fatty liver disease. Hepatology. 2005;41(6):1313-21. 